\title{
Rede Cegonha e seus impactos sobre os nascimentos em um estado do nordeste
}

\section{brasileiro}

\author{
Rede Cegonha and its impacts on births in a region of northeastern Brazil \\ Rede Cegonha y sus impactos en los nacimientos en una región del noreste de Brasil
}

Recebido: 20/02/2021 | Revisado: 28/02/2021 | Aceito: 09/03/2021 | Publicado: 17/03/2021

\author{
Marcela Cristina dos Santos Barros \\ ORCID: https://orcid.org/0000-0001-7863-145X \\ Universidade Federal de Alagoas, Brasil \\ E-mail: mh0673@gmail.com \\ Rita de Cássia Ramires da Silva \\ ORCID: https://orcid.org/0000-0002-0503-4299 \\ Universidade Federal de Alagoas, Brasil \\ E-mail: rita.silva@esenfar.ufal.br \\ Letícia de Macedo Santos \\ ORCID: https://orcid.org/0000-0002-3711-4170 \\ Universidade Federal de Alagoas, Brasil \\ E-mail: leticiademacedosantos@gmail.com \\ Eliza Vitória Nascimento Figueredo \\ ORCID: https://orcid.org/0000-0003-3152-7174 \\ Universidade Federal de Alagoas, Brasil \\ E-mail: eliza.figueredo@eenf.ufal.br \\ Camila Thayná Oliveira dos Santos \\ ORCID: https://orcid.org/0000-0002-9211-4595 \\ Universidade Federal de Alagoas, Brasil \\ E-mail: camila.thay7@gmail.com \\ Alycia Antunes de Carvalho \\ ORCID: https://orcid.org/0000-0002-8753-8158 \\ Universidade Federal de Alagoas, Brasil \\ E-mail: alycia.a.carvalho@gmail.com \\ Thainá da Silva Cabral \\ ORCID: https://orcid.org/0000-0002-6972-2904 \\ Universidade Federal de Alagoas, Brasil \\ thainacabral.cabral@gmail.com \\ Keila Cristina Pereira do Nascimento Oliveira \\ ORCID: https://orcid.org/0000-0003-0167-5889 \\ Universidade Federal de Alagoas, Brasil \\ E-mail: keila.oliveira@eenf.ufal.br \\ Alba Maria Bomfim de França \\ ORCID: https://orcid.org/0000-0001-9474-7137 \\ Universidade Estadual de Ciências da Saúde de Alagoas, Brasil \\ E-mail: alba.franca@uncisal.edu.br \\ Jovânia Marques de Oliveira e Silva \\ ORCID: https://orcid.org/0000-0001-7452-2651 \\ Universidade Federal de Alagoas, Brasil \\ E-mail: jovania.silva@eenf.ufal.br
}

\section{Resumo}

Objetivo: analisar o impacto da Estratégia Rede Cegonha em Alagoas sobre os nascimentos no período de 2011 a 2018. Método: estudo ecológico, de série temporal, com dados secundários oriundos do DATASUS. Os dados foram processados pelos softwares Statistical Package for the Social Sciences. Foram adotadas como variáveis o coeficiente de mortalidade entre os anos de 2011 a 2018, a relação entre a quantidade total de óbitos por causas evitáveis, através de regressão múltipla (p<0,05). Resultados: as taxas apresentaram oscilações, entretanto a rede cegonha potencializou a melhora nos indicadores de morbimortalidade. Observa-se que a maioria dos óbitos fetais poderiam ser evitados por meio de ações de redução das desigualdades sociais, mais consultas de pré-natal de alta qualidade e de melhora na qualidade da atenção obstétrica voltadas à mulher e recém-nascido. Conclusão: evidenciou-se, através da análise dos indicadores, que existe a necessidade de melhoria na atenção à saúde materno-infantil em Alagoas.

Palavras-chave: Saúde materno-infantil; Mortalidade infantil; Indicadores básicos de saúde; Sistemas de informação; Estatísticas vitais. 


\begin{abstract}
Objective: to analyze the impact of the Rede Cegonha Strategy in Alagoas on births from 2011 to 2018. Method: ecological study, time series, with secondary data from DATASUS. The data were processed using the Statistical Package for the Social Sciences software. Variables were the mortality coefficient between the years 2011 to 2018, the relationship between the total number of deaths from preventable causes, through multiple regression ( $\mathrm{p}<0.05)$. Results: the rates understood showed fluctuations, however the Rede Cegonha potentiated the improvements in the indicators of morbidity and mortality. In addition, it was observed that most fetal deaths could be avoided through actions to reduce social inequalities, more high-quality prenatal consultations and to improve the quality of obstetric care aimed at women in the pregnancy-puerperal cycle and to the newborn. Conclusion: it was evident, through the analysis of the indicators, that there is a need for improvement in attention to maternal and child health in Alagoas.
\end{abstract}

Keywords: Maternal and child health; Infant mortality; Health status indicators; Information systems; Vital statistics.

\title{
Resumen
}

Objetivo: analizar el impacto de la Estrategia Rede Cegonha en Alagoas en los nacimientos de 2011 a 2018 . Método: estudio ecológico, serie temporal, con datos secundarios de DATASUS. Los datos se procesaron utilizando el software Statistical Package for the Social Sciences. Las variables fueron el coeficiente de mortalidad entre los años 2011 a 2018 , la relación entre el número total de muertes por causas prevenibles, mediante regresión múltiple ( $\mathrm{p}<0,05)$. Resultados: las tasas entendidas mostraron fluctuaciones, sin embargo la Rede Cegonha incrementó las mejoras en los indicadores de morbilidad y mortalidad. Además, se observó que la mayoría de las muertes fetales podrían evitarse a través de acciones para reducir las desigualdades sociales, consultas prenatales de mayor calidad y mejorar la calidad de la atención obstétrica dirigida a las mujeres en el ciclo embarazo-puerperal y al recién nacido. Conclusión: fue evidente, a través del análisis de los indicadores, que existe la necesidad de mejorar la atención a la salud materno infantil en Alagoas.

Palabras clave: Salud materno-infantil; Mortalidad infantil; Indicadores de salud; Sistemas de información; Estadísticas vitales.

\section{Introdução}

A mortalidade infantil é um fenômeno presente na sociedade, tendo como principais causas: a prematuridade, asfixia perinatal e intraparto, infecções perinatais, malformação congênita e os fatores maternos, sendo os três primeiros destaques mundiais nesse quesito. É válido ressaltar uma proporção considerável de mortes preveníveis decorrente de ações dos serviços de saúde (Carlo \& Travers, 2016).

De acordo com o Fundo das Nações Unidas para Infância - UNICEF (2015), houve um aumento na taxa de nascimentos e redução da taxa de mortalidade neonatal a nível mundial, os dados foram de 36 óbitos por 1.000 Nascidos Vivos - NV em 1990 para 19 em 1.000 NV no ano de 2015, enquanto o número de óbitos neonatais evoluiu de 5,1 milhões para 2,7 milhões. Entretanto, o declínio da mortalidade neonatal no período de 1990-2015 apresentou-se mais lento (47\%) que a pós-neonatal em menores de cinco $\operatorname{anos}(58 \%)$.

Seguindo uma tendência mundial, houve reduções em nível nacional. A média do Brasil, em 2010, foi de 16,0/1.000 NV, entretanto, a região Nordeste do país apresentou coeficientes de 19,1/1.000 NV (Brasil, 2010). E, dentre os estados brasileiros, Alagoas possui a maior Taxa de Mortalidade Infantil (TMI) do país, além de apresentar o segundo pior Índice de Desenvolvimento Infantil (IDI) (Unicef, 2009).

Apesar da melhora na TMI, nacionalmente existem grandes desafios a serem superados para aproximar-se dos níveis das regiões mais desenvolvidas do mundo, com cerca de cinco óbitos para cada mil NV. Apontam-se como entraves que impedem a melhora no índice nacional, dentre outros fatores, as desigualdades regionais, sociais e as iniquidades relacionadas ao nascer no Brasil (Silva, De Moura, Esperidião, Baptista, 2019).

Segundo o Relatório da UNICEF (2015), algumas ações foram fundamentais para a redução da TMI obtida pelo Brasil no período, como o foco na atenção primária em saúde, a melhoria no atendimento materno e ao recém-nascido, a promoção do aleitamento materno, a expansão da imunização e a criação de incentivos de proteção social, como os programas de transferência de renda.

A fim de minimizar as taxas de mortalidades materno-infantil vem sendo desenvolvido no Brasil, há duas décadas, um 
modelo de atenção construído com base na humanização do parto e nascimento. Esse modelo de atenção à saúde da mulher e da criança fomentado pela Rede Cegonha - RC é composto por quatro pilares: (1) Pré-Natal; (2) Parto e Nascimento; (3) Puerpério e Atenção Integral à Saúde da Criança e (4) Sistema Logístico, Transporte Sanitário e Regulação (Brasil, 2011; LNM et al., 2017).

A Rede de Atenção à Saúde Materna e Infantil, implementada em 2011, aponta um novo modelo de atenção à saúde da mulher e da criança, desde o parto até 24 meses após, garantindo o acesso, acolhimento da díade mãe e recém-nascido e um desfecho da gestação com associação à RC. Com a implantação dessas medidas almeja-se a redução da mortalidade materna e infantil, com ênfase no neonato (LNM et al., 2017).

Diante dessas considerações, este trabalho teve como objetivo analisar o impacto da Estratégia Rede Cegonha, em Alagoas, sobre os nascimentos no período de 2011 a 2018. Almeja-se, portanto, a partir deste estudo a abordagem de novas perspectivas capazes de contribuir para a reformulação de políticas públicas em saúde, a fim de atingir uma redução significativa da morbimortalidade materno infantil no estado.

\section{Metodologia}

\subsection{Tipo de estudo}

Trata-se de um estudo ecológico, de série temporal, observacional com a utilização de informações secundárias a partir de dados coletados. O desenho deste estudo possui um componente descritivo para observação das Taxas de Mortalidade Neonatal (TMN), Razão de Mortalidade Materna (RMM), quantidade de NV, casos de sífilis congênita em menores de 1 ano e quantidade de consultas realizadas no Pré-natal em Alagoas no período estudado; e um componente analítico, para análise de óbitos fetais por causas evitáveis, tendência do coeficiente de mortalidade neonatal por causas evitáveis logo após a implementação da Rede cegonha, nas 10 regiões de saúde de Alagoas, por meio da avaliação de impacto com base em dados de adequação (Habicht et al., 1999).

A adequação foi coletada neste estudo considerando que o número de consultas de pré-natal e adequação ao pré-natal indica, dentre outros fatores, o acesso aos sistemas de saúde durante a gestação. Os dados sobre o número de consultas de prénatal, grau de instrução escolar da mãe, idade da mãe e adequação a quantidade de pré-natal foram extraídos do SINASC. As amostras selecionadas estão relacionadas aos NV e a mortalidade, no período de 2011 a 2018, por residência da mãe. 2.2. Coleta de dados

As coletas de dados realizadas a partir dos Sistemas de Informações sobre Nascidos Vivos (SINASC), Sistema de Informação de Mortalidade (SIM) no site do Departamento de informática do SUS (DATASUS) e dados de domínio público do Departamento de Doenças Crônicas e Infecções Sexualmente Transmissíveis do MS. Os dados coletados são referentes ao período de janeiro de 2011 a dezembro de 2018.

No Sistema de Informação e Mortalidade (SIM), foram selecionadas as informações sobre mortalidade neonatal por causas evitáveis, óbitos fetais segundo o tipo de parto e óbitos maternos. Os óbitos infantis (em menores de um ano) foram categorizados em: mortalidade neonatal precoce (óbitos ocorridos de zero a seis dias de vida), mortalidade neonatal tardia (de sete a 27 dias de vida) e mortalidade pós-neonatal (de 28 dias a um ano de vida) (Brasil, 2009).

\subsection{Procedimentos de análise de dados}

Após a consulta às informações nos sistemas, foram feitas tabulações no Microsoft Excel 2010 que, posteriormente, foram exportadas para o programa softwares StatisticalPackage for the Social Sciences (SPSS) para o processamento dos dados tabulados. 
Os resultados foram apresentados de acordo com os indicadores-chave para a implementação da Rede Cegonha: percentual de gestantes que realizaram sete ou mais consultas pré-natais e a consulta de puerpério; número de NV e as taxas dos tipos de parto (importantes indicadores de atenção à saúde); as taxas de óbitos infantis e o número absoluto de óbitos maternos por faixa etária (importantes indicadores de mortalidade) (Brasil, 2011).

Para observar se houve redução da tendência do coeficiente de mortalidade neonatal por causas evitáveis, foram calculadas as variações percentuais anuais (APC) através da modelagem pelo método de regressão joinpoint. Trata-se de um método de análise de tendências temporais, no qual o valor significativo referente à redução nesse estudo foi de APC $<1$ (redução); APC=0 (nula); APC>1 (aumento) (De Araujo \& De Andrade, 2019).

Avaliou-se a relação entre a quantidade total de óbitos por causas evitáveis, através de regressão múltipla. Neste método estabeleceu-se como variável dependente: causas evitáveis de óbitos; variável independente: reduzíveis pelas ações de imunização; reduzíveis por atenção à mulher na gestação; reduzíveis por adequada atenção à mulher no parto; reduzíveis por adequada atenção ao recém-nascido; reduzíveis por ações adequadas de diagnóstico e tratamento; reduzíveis por ações adequadas de promoção da saúde, vinculadas a ações de atenção à saúde; causas mal definidas e demais causas (não claramente evitáveis). Foram considerados estatisticamente significantes o valor de $(\mathrm{p}<0,05)$.

Utilizou-se, ainda, $t$ student, que consiste em um método de hipótese que avalia se há diferença significativa entre as médias de duas amostras, para avaliação do impacto do ano de 2016. Para avaliação do número de consultas durante o pré-natal, comparou-se a média de consultas realizadas antes de 2016 e após o ano de 2016 . Valores de $(p<0,05)$ foram considerados estatisticamente significantes.

\subsection{Aspectos éticos}

Por envolver somente análise de dados secundários de domínio público não foi necessário o encaminhamento para apreciação e autorização do Comitê de Ética em Pesquisa (CEP) da Universidade Federal de Alagoas - UFAL.

\section{Resultados}

O banco de dados do SIM registrou 6.080 óbitos em menores de um ano de mães residentes em Alagoas no período de 2011 a 2018. O SINASC, por sua vez, registrou 414.423 NV no período analisado. Com TMI de 14,67/1000 NV, média do TMI no período mencionado. Em 2011, observou-se um CMI de 18,99/1000 NV, com um recuo nos anos de 2016, 2017 e 2018, com taxas de $14,30 / 1000 \mathrm{NV}, 13,40 / 1000 \mathrm{NV}$ e $12,53 / 1000 \mathrm{NV}$, respectivamente.

De 2011 a 2018 foram registrados 414.423 NV e 1.983 óbitos por causas evitáveis em menores de um ano (período pósneonatal) em Alagoas, dos quais 928 (13,07\%) ocorreram no período neonatal tardio e, destes, a maioria 3.169 (44\%) constituída por óbitos neonatal precoce.

A análise da quantidade total de óbitos pelo método joinpoint representado na Figura 1 apresentou uma queda entre 2011 e 2018, com pontos de inflexão. 
Figura 1 - Tendência do coeficiente de mortalidade neonatal por causas evitáveis, pelo método joinpoint no período de 2011 a 2018, Alagoas, Nordeste, Brasil.

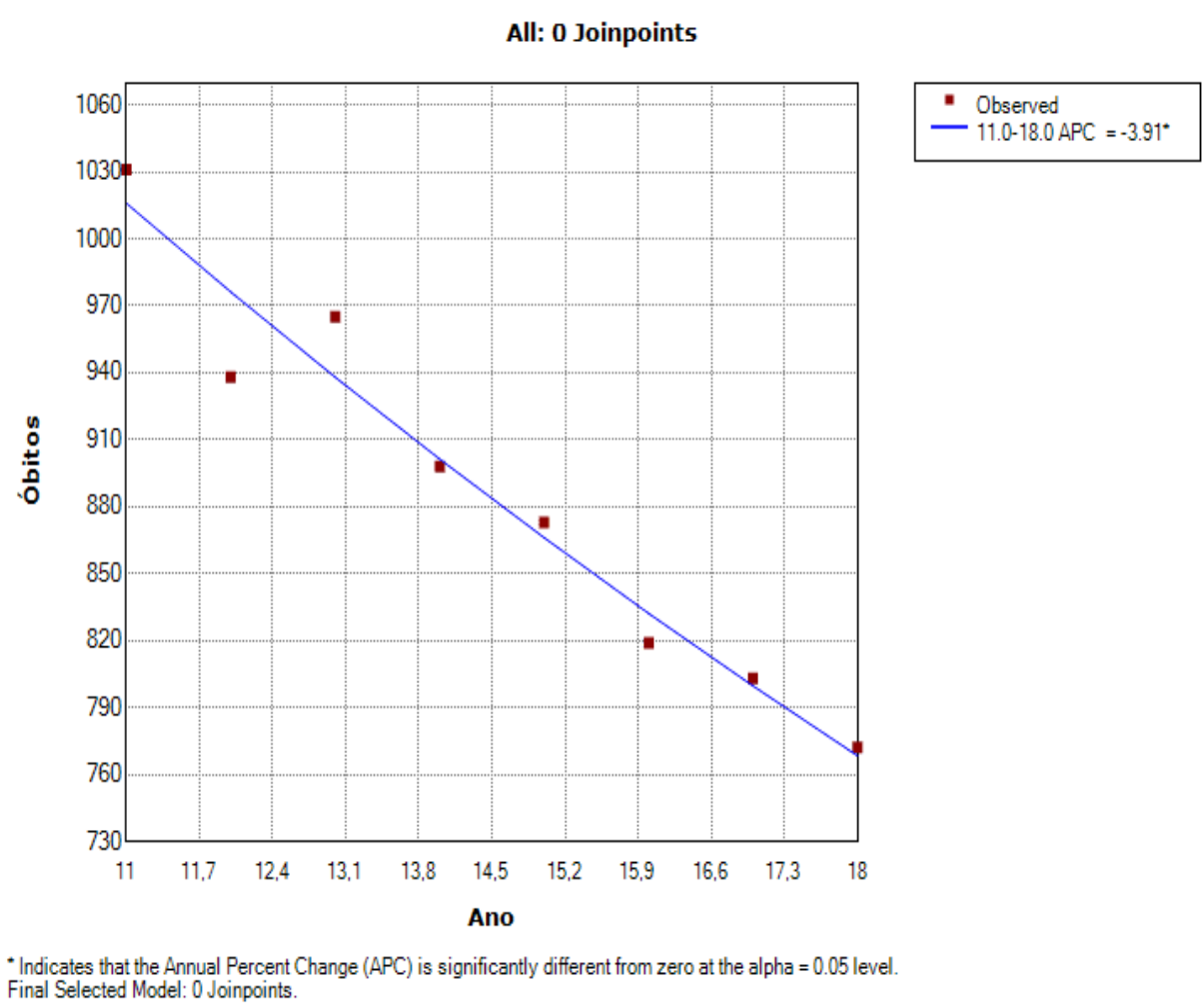

Legenda: *Indica que a mudança percentual anual é significativamente diferente de zero no nível alfa $=0,05$.

Modelo final selecionado: 0 pontos de junção.

Fonte: Autores.

Em Alagoas, ao todo foram 414.422 atendimentos de consulta de pré-natal entre os anos de 2011 e 2018 , em todas as 10 regiões de saúde do estado de Alagoas. A partir do ano de 2016 houve um aumento na quantidade de gestantes que realizaram 7 consultas ou mais na região nordeste. É válido ressaltar que o ano de 2016 foi um marco e, após este, todas as quantidades de consultas variaram de forma significativa. Por meio de análise, infere-se que as gestantes passaram a ter mais consultas de prénatal $(\mathrm{p}$ valor $=0,041)$.

Constatou-se através da análise que mães que possuem 8 a 11 anos de instrução escolar realizaram um pré-natal "mais que adequado", correspondendo a 64,066. Em contrapartida, das que possuíam 12 anos ou mais de instrução, apenas 20.668 realizam o pré-natal "mais que adequado".

A Figura 2 apresenta dados relativos aos casos de SC, que foram registrados entre os anos de 2011 e 2018 . Constatouse um pico de 440 casos em 2018, com taxa de detecção de 8,7/1000NV. Observou-se que desde a implantação da RC, os casos de SC diminuíram a partir de 2013. Entretanto, obteve-se um aumento expressivo na taxa de detecção da SC, com taxas de 7,8(2013); 8,0(2014); 7,4(2015); e em 2016 taxa de 6,6/1000NV quando comparado ao ano de 2009, com taxa de detecção de $3,2 / 1000 \mathrm{NV}$. 
Figura 2 - Casos de sífilis congênita em menores de um ano de idade no período de 2011 a 2018, Alagoas, Nordeste, Brasil.

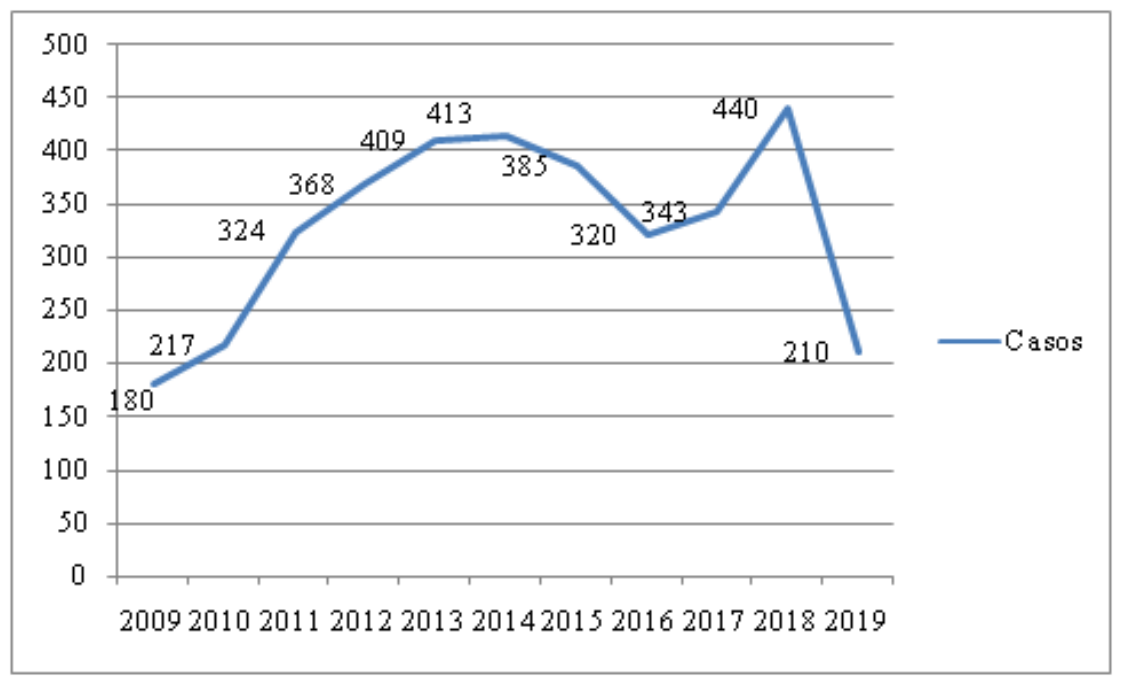

Fonte: Autores.

Observa-se, conforme a Tabela 1, que no período de 2011 a 2018, foram registrados 234 óbitos maternos em Alagoas, resultando em uma taxa de mortalidade de 56,46 óbitos para cada 100.000 NV. A maior taxa registrada em 8 anos se deu em 2014, com 104,12/100.000 NV, havendo uma tendência à redução, uma vez que, em 2015, a Razão de mortalidade materna (RMM) foi de 57,40/100.000 NV. Observa-se que, para cada ano, houve oscilações na RMM. Em 2017 houve uma queda significativa da RMM, com valor de 32,21 (6,83\%), sendo a menor taxa de RMM desde 2011.

Tabela 1 - Coeficiente de mortalidade materna a cada 100.000 nascidos vivos no período de 2011 a 2018, Alagoas, Nordeste,

Brasil.

\begin{tabular}{llll}
\hline & Óbitos maternos & RMM \\
\hline ANO & $\mathbf{n}$ & $\%$ & \\
\hline 2011 & 28 & $11,96 \%$ & 51,58 \\
2012 & 24 & $10,25 \%$ & 44,21 \\
2013 & 31 & $13,24 \%$ & 59,06 \\
2014 & 54 & $23,07 \%$ & 104,12 \\
2015 & 30 & $12,82 \%$ & 57,40 \\
2016 & 25 & $10,68 \%$ & 51,90 \\
2017 & 16 & $6,83 \%$ & 32,21 \\
2018 & 26 & $11,11 \%$ & 49,52 \\
TOTAL & 234 & 100 & 56,46 \\
\hline
\end{tabular}

Fonte: Autores.

A correlação entre o tipo de parto e óbitos fetais evidencia que morreram mais fetos por via vaginal, do que por parto cesáreo, e, ainda, que houve um declínio constante no número de óbitos fetais por via vaginal em 2016. Nos anos anteriores, entre 2000 e 2010, a soma dos óbitos anuais por via vaginal era de 5.680, após a implantação da RC, houve uma redução de até 
42,71\% (representa 3.254 casos) nos casos de óbitos fetais por via vaginal. A mesma trajetória ocorreu com os óbitos por parto cesáreo, em que houve declínio de $12,87 \%$.

Por análise de regressão há significância estatística, quando o p-valor encontrado é menor do que o definido para o estudo. Observou-se valores significativos quanto causas múltiplas à atenção à mulher no parto (p=0,011), ações de promoção à saúde vinculadas a ações de atenção $(\mathrm{p}=0,024)$ e causas mal definidas $(\mathrm{p}=0,01)$.

A seguir, consta na Tabela 2, as relações de distribuição de recursos, profissionais e leitos para a rede de saúde maternoinfantil, no período estudado. Houve um significativo aumento das razões, quando em comparação ao ano (2011) da implantação da RC. Com destaque para o fornecimento de enfermeiro-obstetra/100.000 hab., que passou de r: 3,58 em 2011 para r: 7,64 em 2018.

Tabela 2 - Oferta de recursos, profissionais e leitos no período de 2011 a 2018, Alagoas, Nordeste, Brasil.

\begin{tabular}{|c|c|c|c|}
\hline & Indicador & 2011 & 2018 \\
\hline mil habi & $\begin{array}{l}\text { Razão ginecologista-obstetra por } 100 \\
\text { antes }\end{array}$ & 17,44 & 22,91 \\
\hline habitant & $\begin{array}{l}\text { Razão enfermeiro-obstetra por } 100 \text { mil } \\
\text { s }\end{array}$ & 3,58 & 7,64 \\
\hline \multirow{3}{*}{ vivos } & Leitos obstétricos por mil habitantes & 1,00 & 0,74 \\
\hline & Recém Nato Normal por mil nascidos & 0,79 & 0,80 \\
\hline & RN Patológico por mil nascidos vivos & 0,50 & 0,86 \\
\hline vivos & Conjunto para $\mathrm{RN}$ por mil nascidos & 9,19 & 7,73 \\
\hline
\end{tabular}

Fonte: Autores.

\section{Discussão}

Embora o Coeficiente de Mortalidade Infantil - CMI em Alagoas esteja maior que o nacional, Alagoas apresentou melhores resultados na redução de mortalidade materna. Com isso, infere-se que a saúde da mulher tem sido priorizada em detrimento da atenção à saúde do recém-nascido.

De acordo com Mariano e Marta (2018), estados como Norte e Nordeste possuem, historicamente, os menores Índices de Desenvolvimento Humano - IDH e os maiores índices de CMI do país. Ainda em 2011, no estado de Alagoas, o CMI foi de 18,99\%/1000NV comparando ao estado do Piauí situado na região Nordeste, por exemplo, obteve o CMI de 16,9\%/1000 NV, no mesmo ano. Entretanto, ainda assim, persistem as disparidades ao observar as informações para as regiões, estados e municípios (Mariano \& Marta, 2018).

Durante análise das taxas ao longo dos anos, nota-se que houve um importante recuo do CMI em Alagoas nos anos de 2016, 2017, 2018, porém evidencia-se que a taxa ainda está acima da média nacional que, em 2018, foi de 12,4\%/1000 NV (IBGE, 2018), comparado ao ano de 2017 em Alagoas, que foi de 13,40\%/1000 NV.

Identificou-se que entre os anos de 2011 e 2018, os óbitos neonatais ocorreram em sua maioria no período de 0 a 6 dias, sendo considerado um óbito neonatal precoce 3.169 (44\%). Esta informação corrobora com outros estudos (Brasil, 2014). em que a ocorrência prevalente dos óbitos no período neonatal precoce, chega a cerca de 53,0\% dos óbitos infantis, com destaque para os óbitos ocorridos nas primeiras 24 horas de vida, acompanhando a característica dos países “em desenvolvimento".

Em consonância com esses dados, Teixeira e autores parceiros (2019) relacionam como as três principais causas da 
mortalidade neonatal, sendo: síndrome da angústia respiratória, imaturidade extrema e muito baixo peso ao nascer, são classificadas como causas evitáveis quando é proporcionado uma adequada atenção na gestação.

Utilizou-se o método joinpoint, após o uso desse método, foi identificada uma queda entre 2011 e 2018 , com pontos de inflexão, conforme o exposto no Figura 1.

Em um estudo realizado recentemente para avaliar o impacto da Rede Cegonha no Coeficiente de mortalidade neonatal (CMN) evitável, foi observado, ao utilizar o método de regressão joinpoint, que houve redução acentuada destes coeficientes, particularmente, o Coeficiente de Mortalidade Neonatal Precoce (CMNP), assemelhando aos dados deste estudo (Figura 1). Houve também uma redução expressiva do CMN após a implantação da RC. Sendo assim, a análise mostrou que os CMN evitáveis apresentaram acentuada tendência de queda no Estado de Pernambuco (de Lima et al., 2020). Sendo um importante indicador relacionado à atenção em saúde durante o parto e puerpério.

Houve um aumento significativo a partir do ano de 2016 nas gestantes que realizaram 7 consultas ou mais no estado. Este momento ocorre devido à criação do PES 2016-2019, que intensificou as ações da RC no âmbito da Rede de saúde materno infantil em Alagoas. O plano explicitou os compromissos do governo para a saúde dos alagoanos e foi construído, a partir da análise situacional e do perfil epidemiológico, com base nas necessidades de saúde da população do estado de Alagoas (CONASS, 2016).

Em outras pesquisas de abrangência nacional, a adequação do pré-natal se destacou em mulheres com um grau de instrução maior (Domingues et al., 2015). A escolaridade é um fator de análise, visto que as mulheres que possuíam até 8 anos de escolaridade, geralmente, realizavam menos pré-natais. Fato este que pode relacionar a menor taxa de escolaridade aos empregos de baixa remuneração e carga horária excessiva, assim, as mulheres trabalhavam mais e não compareciam aos serviços de saúde.

No que se refere ao pré-natal, Sífilis Congênita - SC é uma doença importante que deve ser observada, pelo fato ser um indicador sentinela da qualidade prestada no pré-natal. Por meio desta vigilância, será possível medir esforços para desfechos favoráveis (Oliveira, Felix, Souza, Machado, 2019). A SC constitui um grave problema de saúde pública, trata-se de uma doença de evolução crônica e de abrangência mundial (Brasil, 2018). O conhecimento precoce da SC possibilita o tratamento em tempo oportuno e redução de sequelas ao RN.

Em Alagoas, com meta de controle do crescimento da curva, a Programação Estadual de Saúde (PES) buscou uma redução de casos em 10\% no período de 2016-2019. Concomitantemente, a Programação Anual de Saúde (PAS) 2018 do estado também estabeleceu como meta uma redução de 2,5\% na incidência de SC (Alagoas, 2018).

Em um estudo realizado recentemente, que teve por objetivo avaliar a taxa de notificação da SC em Alagoas, identificouse que o estado de Alagoas continua distante de alcançar a meta da Organização Pan-Americana da Saúde (OPAS) de eliminação da SC como problema de saúde pública (Oliveira, Felix, Souza, Machado, 2019).

Nesse estudo, evidenciou-se uma grande oscilação na RMM em Alagoas. A RMM em Alagoas, correspondente ao período de 2011 a 2018, é de 56,46/100.000 NV. No município de Minas Gerais, os resultados se assemelham aos encontrados nesta pesquisa, pela tendência à redução da RMM, quando, entre 2005 e 2015, ela foi de 71.054/100.000 NV (Martins \& Silva, 2018). As RMM podem estar associadas a ausência de cuidados necessários, boas práticas e cuidados obstétricos adequados.

Para Martins e colaboradores (2019), a mortalidade materna permanece como um grande problema de saúde pública, sendo um importante indicador de saúde da mulher.

Em Alagoas, os óbitos maternos por causas obstétricas diretas chegaram a 73,50\%, entre 2011 e 2018, corroborando com estudos realizados em outro estado brasileiro, como Bahia $(62,8 \%)$, este grupo relaciona-se às gestantes com maior vulnerabilidade social e que não tiveram uma adequada assistência na gestação (Carvalho et al., 2016).

Com a implantação da RC entre os anos de 2011 e 2012, buscou-se a qualificação do cuidado materno-infantil no Brasil, 
concomitante, objetivou-se a redução dos óbitos maternos, esperava-se, portanto, uma redução temporal da mortalidade materna no estado de Alagoas (De La Torre et al., 2018). Entretanto, estudos revelam que Alagoas não apresentou mudança temporal (2010-2016) da mortalidade (Duarte et al., 2020), embora a taxa tenha reduzido insuficientemente a RMM de 51,58/100.000 NV (2011) para 49, 52 (2018).

Em um estudo sobre o perfil epidemiológico da mortalidade materna, autores (Martins \& Silva, 2018) justificam os altos índices de óbito materno as altas taxas de cesariana. Em Alagoas, a taxa de cesarianas compreende o percentual de 54,72\%, o que poderia justificar o número de óbitos no período estudado. Nesse mesmo estudo, a cesárea foi realizada em $38,8 \%$ dos partos. Autores ainda afirmam que, o nascimento por cesárea, comparado ao parto vaginal, está relacionada a mortalidade materna e neonatal (Diniz et al., 2016).

O Ministério da Saúde, em 2015, apresentou dados referentes a realização do parto cesáreo e normal, com percentuais de $40 \%$ e $84 \%$, respectivamente, (Eckstein, 2015). Além da taxa de cesáreas, o óbito fetal é um indicador de saúde que deve ser avaliado para analisar qual o tipo de assistência obstétrica que está sendo oferecida em todo o processo gestacional (Jeronimo Lima et al., 2017).

Neste estudo, em relação ao óbito relacionado ao tipo de parto, destacou-se, com maior prevalência o parto vaginal (63, $89 \%$ ) em relação à cesariana (28,96\%). Em um estudo recente, foi observado que, ao realizar a análise de dados dessa natureza, deve-se levar em ponderação que, quando o feto é natimorto e, quando o diagnóstico é feito intraútero, a via de parto preferencial é a normal (Giraldi et al., 2019) Tal fato pode explicar a crescentes taxas nos óbitos por via vaginal encontradas neste estudo.

O mesmo resultado foi encontrado no estudo de Silva (2020), em um estudo realizado no estado do maranhão, no qual houve um alto percentual de casos de óbitos fetais relacionados ao parto vaginal (64\%). Ainda assim, afirma Barros (2019) que os partos cesáreos estão associados ao risco de morbimortalidade materna e neonatal em curto e longo prazo.

A Rede Cegonha representa avanços significativos, refletidos no aumento do acesso aos serviços de saúde e na redução da taxa de mortalidade infantil, no entanto a abordagem biologicista e mecanizada nos serviços de saúde se colocam como entraves à melhoria de indicadores em saúde (Backes et al., 2019). Além de proporcionar boas práticas no parto que favorecem o incentivo ao protagonismo da parturiente (Feijão et al., 2017).

No que se refere à oferta de recursos humanos e físicos (Tabela 2), conforme preconizado pelo MS (r: 0,28 por mil habitantes), a quantidade encontrada neste estudo denota que os leitos estão devidamente distribuídos, pois se observa uma r: 1,0/1000 hab. (2011), e em 2018 r: 0,74. Esses achados se identificam com estudos de Lima et al. (2020), que demonstrou r: 0,28/1000 hab. (De Lima et al., 2020).

\section{Conclusão}

A análise temporal contida neste artigo traz o panorama da redução de mortalidade em um estado com a maior taxa da federação, relacionando os índices às condições de saúde regionais, às desigualdades sociais, e às condições de vida e trabalho de mães alagoanas. Verificou-se neste estudo um declínio do Coeficiente de Mortalidade Infantil no período estudado em Alagoas, apesar de ainda se encontrar acima da média nacional. O número de óbitos neonatais precoces foram os mais registrados, os quais poderiam ter sido evitados, através de ações adequadas em ações de promoção à saúde à grávida e ao recém-nascido.

Após a divulgação do PES 2016-2019, a quantidade de gestantes que realizaram 7 ou mais consultas na região nordeste aumentou, fato importante que se contrapõe ao ano 2000 em que o acesso a esses serviços era reduzido. Ainda, constatou-se que os óbitos fetais se sobressaíram no parto vaginal em comparação ao parto cesáreo, apesar das altas taxas de cesáreas e da alta mortalidade materna.

Diante da metodologia proposta, percebe-se que o trabalho alcançou os dados necessários para análise do impacto da 
Rede Cegonha. Entretanto, essa pesquisa observacional utiliza como fonte dados secundários, assim, dentre as limitações da fase de coleta e análise de dados podem-se elencar a subnotificação e a possível desatualização dos dados brutos coletados, com ênfase nos dados mais antigos.

O trabalho aponta os avanços alcançados e as dificuldades, ainda, existentes na qualidade do atendimento prestado às gestantes e puérperas, contribuindo, portanto, como subsídio e incitação a novos estudos na área, na construção e no melhoramento das políticas públicas em saúde enfatizando o acesso, resolutividade e acolhimento na assistência prestada em Alagoas.

\section{Referências}

Alagoas, Secretaria de Estado da Saúde. (2018). Programação Anual de saúde.

Backes, D. S., Pereira, S. B., Marchiori, M. R. C. T., Pilecco, J. C., Backes, M. T. S., \& Moreschi, C. (2019). Construção e validação de construto de boas práticas de atenção ao parto/nascimento. Enfermagem Em Foco, 10(5), 85-90. https://doi.org/10.21675/2357-707x.2019.v10.n5.2593.

Barros, P. de S., de Aquino, É. C., \& de Souza, M. R. (2019). Mortalidade fetal e os desafios para a atenção à saúde da mulher no Brasil. Revista de Saude Publica, 53(1). https://doi.org/10.11606/S1518-8787.2019053000714.

Brasil, Instituto Brasileiro de Geografia e Estatística. (2018). Estatísticas vitais-Brasil: 2011-2018.

Brasil, Ministério da Saúde. (2010). Estatísticas vitais - mortalidade e nascidos vivos, DATASUS.

Brasil, Ministério da Saúde (2011). Portaria No 1.459, de 24 de junho de 2011.

Brasil, Ministério da Saúde. (2014). Saúde Brasil 2013: uma análise da situação de saúde e das doenças transmissíveis relacionadas à pobreza, Secretaria de Vigilância em Saúde, Departamento de Análise de Situação em Saúde, Brasília.

Brasil, Ministério da Saúde. (2018). Boletim Epidemiológico de Sífilis / Secretaria de Vigilância em Saúde, Brasília.

Brasil, Secretaria de Atenção à Saúde (2009). Manual dos Comitês de Mortalidade Materna / Departamento de Ações Programáticas Estratégicas, 3. ed. Brasília: O Ministério.

Carlo, W. A., \& Travers, C. P. (2016). Maternal and neonatal mortality: time to act. Jornal de Pediatria (Versão Em Português), 92(6), 543-545. https://doi.org/10.1016/j.jpedp.2016.08.001.

Carvalho, L. R. de O., Fonseca, L. M. L., Coelho, M. T. C., Machado, M. G. de S., Carvalho, M. B. de, \& Vidal, C. E. L. (2016). Mortalidade de mulheres em idade fértil entre 1998 e 2012 na microrregião de Barbacena. Rev. Interdisciplin. Estud. Exp. Anim. Hum. (Impr.), 8(único), 15-22.

Conselho Nacional de Secretários de Saúde, CONASS. (2016). Plano Estadual de Saúde 2016-2019.

De Araújo, I. C. F. G., dos Santos Ferreira, T. L., de Araújo, D. V., Melo, K. D. F., \& de Andrade, F. B. (2019). Qualidade do parto e impacto nos indicadores da saúde da criança. Revista Ciência Plural, 5(1), 18-33.

De La Torre, A., Nikoloski, Z., \& Mossialos, E. (2018). Equidade de acesso às intervenções de saúde materna no Brasil e na Colômbia: um estudo retrospectivo. Internacional Journal for Equity in Health, 17(43). https://doi.org/10.1186/s12939-018-0752-x.

De Lima, S. S., Braga, M. C., de Moraes Vanderlei, L. C., Luna, C. F., \& Frias, P. G. (2020). Avaliação do impacto de programas de assistência pré-natal, parto e ao recém-nascido nas mortes neonatais evitáveis em Pernambuco, Brasil: estudo de adequação. Cadernos de Saude Publica, 36(2). https://doi.org/10.1590/0102-311X00039719.

Martins, A. C. S., \& Silva, L. S. (2018). Perfil epidemiológico de mortalidade materna. Revista Brasileira de Enfermagem, 71, 677-683.

Diniz, C. S. G., Miranda, M. J., Queiroz, J. R., Queiroz, M. R., Salgado, H. O. (2016). Why do women in the private sector have shorter pregnancies in Brazil? Left shift of gestational age, caesarean section and inversion of the expected disparity. J.Hum.Growth Dev., 26(1):33-40. https://doi.org/107322/jhgd.113712.

Domingues, R. M. S. M., Viellas, E.F., Dias, M. A. B., Torres, J. A., Filha, M. M. T., Gama, S. G. N., Leal, M. C. (2015). Adequação da assistência pré-natal segundo as características maternas no Brasil. Cad. Saúde Pública, 37(3).

Duarte, E. M. da S., Machado, M. F., Alencar, É. T. D. S., de Araújo, M. D. P., da Fonseca, L. G. A., Correia, D. S., da Silva, S. M., \& de Souza, C. D. F. (2020). Mortalidade materna e vulnerabilidade social no Estado de Alagoas no Nordeste brasileiro: uma abordagem espaço-temporal. Revista Brasileira de Saude Materno Infantil, 20(2), 575-586. https://doi.org/10.1590/1806-93042020000200014.

Eckstein, I. (2015). Ministério da Saúde e ANS publicam regras para estimular parto normal na saúde suplementar, Brasília.

Feijão, L. B. V., Boeckmann, L. M. M., \& Melo, M. C. (2017). Conhecimento De Enfermeiras Residentes Acerca Das Boas Práticas Na Atenção Ao Parto. Enfermagem Em Foco, 8(3), 35-39. https://doi.org/10.21675/2357-707x.2017.v8.n3.1318. 
Research, Society and Development, v. 10, n. 3, e30810313166, 2021

(CC BY 4.0) | ISSN 2525-3409 | DOI: http://dx.doi.org/10.33448/rsd-v10i3.13166

Giraldi, L. M., Corrêa, T. R. K., Schuelter-Trevisol, F., \& Gonçalves, C. O. (2019). Óbito fetal: fatores obstétricos, placentários e necroscópicos fetais/Fetal death: obstetric, placental and fetal necroscopic factors. Jornal Brasileiro de Patologia e Medicina Laboratorial, 55(1), 109-113. https://doi.org/10.5935/16762444.20190007.

Habicht, J. P., Victora, C. G., \& Vaughan, J. P. (1999). Evaluation designs for adequacy, plausibility and probability of public health programme performance and impact. International Journal of Epidemiology, 28(1), 10-18. https://doi.org/10.1093/ije/28.1.10.

Jeronimo Lima, K., Soares Chaves, C., de Oliveira Gomes, E., Aparecida de Lima, M., Cristina Pascôa Candeira, E., Kécia Silevira Teófilo, F., Pereira Nunes, G., \& Alves Alencar Viana, R. (2017). Análise da situação em saúde: a mortalidade fetal na 10ª região de saúde do Ceará. Revista Brasileira Em Promoção Da Saúde, 30(1), 30-37. https://doi.org/10.5020/18061230.2017.p30.

LNM, S., Silveira, A. P. K. F., \& Morais, F. R. R. (2017). Programa de Humanização do Parto e Nascimento: aspectos institucionais na qualidade da assistência. Rev Enferm UFPE on Line., 11(8), 3295-3302. https://doi.org/10.5205/reuol.11135-99435-1-ED.1108sup201713.

Martins, A. C. S., \& Silva, L. S. (2018). Epidemiological profile of maternal mortality. Revista Brasileira de Enfermagem, 71, 677-683. https://doi.org/10.1590/0034-7167-2017-0624.

Mariano, J. L., \& Marta, F. (2018). Eficiência na redução da mortalidade infantil: uma análise para os municípios da região Nordeste.

Oliveira, E. M. De, \& Celento, D. D. (2016). A temática da Rede Cegonha. Revista de Saúde, 07(1), 33-38.

Oliveira, T. F., Felix, I. C. G., Souza, C. D. F., Machado, M. F. (2019). Perfil epidemiológico da sífilis congênita em alagoas (2008-2017). Saúde Meio Ambient. 8:237-247.

Silva, L. S. R. da, Silva, P. S. R. da, Santos, M. C. A., Silva, A. A., Oliveira, L. S., Silva, M. B. P. da, Varão, A. C. de A., Paiva, M. S. B., Rodrigues, R. L., \& Cardoso, L. S. P. (2020). Perfil sociodemográfico e obstétrico dos óbitos fetais de gestantes residentes em um município do estado do Maranhão. Revista Eletrônica Acervo Saúde, 45. https://doi.org/10.25248/reas.e3113.2020.

Silva, V. A; De Moura, F. R.; Esperidião, F.; Baptista, C. H. M. S. (2019). Desigualdades socioeconômicas: uma análise sobre os determinantes da tax a de mortalidade infantil nos municípios brasileiros. Revista Brasileira de Estudos Regionais e Urbanos, 13(1), 73-97.

Teixeira, J. A. M., Araujo, W. R. M., Maranhão, A. G. K., Cortez-Escalante, J. J., Rezende, L. F. M. de, \& Matijasevich, A. (2019). Mortalidade no primeiro dia de vida: tendências, causas de óbito e evitabilidade em oito Unidades da Federação brasileira, entre 2010 e 2015. Epidemiologia e Servicos de Saude : Revista Do Sistema Unico de Saude Do Brasil, 28(1). https://doi.org/10.5123/S1679-49742019000100006.

UNICEF, United Nations Children's Fund. (2009). Situação Mundial da Infância 2009: Saúde Materna e Neonatal. Brasília- DF.

UNICEF. (2015). Levels \& Trends in Child Mortality: Report 2015 - Estimates Developed by the UN Inter-agency Group for Child Mortality Estimation. 